\title{
Pedagogía crítica, la visión para favorecer la autonomía en el alumno
}

\section{Critical pedagogy, the visión to promote studen autonomy}

\author{
CHÁVEZ-LÓPEZ, María Margarita†* \& MAGDALENO-CASTRO, Benigno
}

Centro Pedagógico de Durango

ID $1^{\mathrm{er}}$ Autor: María Margarita, Chávez-López / ORC ID: 0000-0002-6642-2455

ID $1^{\mathrm{er}}$ Coautor: Benigno, Magdaleno-Castro / ORC ID: 0000-0002-1404-6136

DOI: $10.35429 /$ JCP.2019.9.3.21.28

Recibido 03 de Junio, 2019; Aceptado 30 de Septiembre, 2019

\section{Resumen}

Las múltiples dificultades que se viven dentro del aula son reflejo de la realidad de alumnos, maestros y comunidad escolar. Mucho se habla de la problemáticas de los alumnos, sus conductas, la escasa consolidación de aprendizajes, falta de participación, su vulnerabilidad ante el contexto en que viven, sin embargo muy pocas veces el docente asume alguna de éstas como propias y busca darles solución a través de su práctica. El presente es el seguimiento de un docente investigador que como propósito se planteó la transformación de su práctica para favorecer el pensamiento crítico en alumnos de nivel primaria y docentes en formación. Para ello se utilizó el paradigma sociocrítico bajo un enfoque de investigación-acción, las acciones realizadas tuvieron como base la pedagogía crítica. Se encontró que la pedagogía crítica mejora la práctica docente, propiciando la trasformación de la realidad de los alumnos a través del desarrollo del pensamiento crítico favoreciendo la autonomía en los alumnos.

Pedagogía crítica, Investigación acción, Alumno autónomo

\begin{abstract}
The multiple difficulties that are experienced within the classroom are a reflection of the reality of students, teachers and the school community. There is much talk about the problems of the students, their behaviors, the poor consolidation of learning, lack of participation, their vulnerability to the context in which they live, however rarely does the teacher assume any of these as their own and seeks to solve them through their practice. The present is the follow-up of a research teacher whose purpose was the transformation of his practice to favor critical thinking in elementary students and teachers in training. For this, the socio-critical paradigm was used under an action research approach, the actions carried out were based on critical pedagogy. It was found that critical pedagogy improves the teaching practice, promoting the transformation of students' reality through the development of critical thinking, favoring autonomy in students.
\end{abstract}

Critical pedagogy, Action research, Autonomous student

\footnotetext{
* Correspondencia del Autor (mm.chavez.lopez@gmail.com)

$\dagger$ Investigador contribuyendo como primer autor.
} 


\section{Introducción}

A lo largo de la historia, las formas de enseñanza han evolucionado, con ellos el proceder del docente en su quehacer cotidiano. Independientemente del estilo de enseñanza o el método utilizado, los docentes tienen claro un fin común, propiciar las condiciones necesarias para que el alumno aprenda. Por ello, toda acción que es vista como inadecuada es meramente subjetiva pues cada método o modelo existente sigue vigente por una razón, porque ha sido un factor de cambio en la realidad en la que surgió.

Dentro de las aulas, los maestros desempeñan sus acciones con base a lo aprendido durante su formación como docentes, procuran innovar aplicando estrategias que observan funcionaron con compañeros, olvidando en ocasiones que para diseñar las acciones a ejecutar en su grupo deben considerar las características de éste, así como las de su propia práctica.

Pese al cumulo de estrategias, métodos, modelos, pareciera que hay momentos en que los docentes proceden de manera mecánica repitiendo los mismos vicios, mismas acciones e inclusive mismas planificaciones para los distintos grupos que pudiesen atender pues argumentan su efectividad en los grupos. Basta la falla de la dinámica aplicada por el docente con un grupo para pensar que es el grupo el que presenta el problema, sin analizar que probablemente sea su práctica o bien que asumiéndolo como propio podría mejorar la realidad del grupo.

Durante los Consejos Técnicos Escolares (CTE), los docentes expresan cada una de las dificultades, éxitos y retos que se van presentando siempre con la intención de mejorar. Sin embargo, en muchas de las ocasiones se han convertido en espacio de incertidumbre y duda, pues existen maestros que adjudican a los estudiantes los problemas del grupo en general, que van desde las cuestiones contextuales hasta conductuales.
Generalmente se justifican los resultados de los alumnos, así como las como las dificultades para aprender con las deficiencias que ellos presentan, tales como falta de comprensión lectora, bajo dominio de contenidos, dificultad en la convivencia escolar, falta de consolidación de aprendizajes, o bien, con la poca participación de los padres de familia así como los contextos poco favorecedores para el aprendizaje. Sin embargo, si esa es la realidad de muchas escuelas y cada CTE expresan las mismas dificultades pese al cambio del ciclo escolar, entonces qué está fallando.

Bajo a dichas premisas, surge la interrogante que orientara esta investigación ¿qué hacer con las distintas dificultades que surgen dentro del aula que sesgan la consolidación de aprendizajes y modificación de la conducta de los alumnos?, con base en este cuestionamiento se comprendió que el análisis debía centrarse en la práctica del docente más que en las problemáticas de los alumnos.

Estudiar la práctica docente permite identificar los problemas reales de la educación en las aulas, por ello, que el análisis sobre ésta sea una razón de peso para conocerlas y buscar mejorarlas. Esto sugiere que el docente ponga en juego cada una de las herramientas, habilidades y conocimientos que le permitan atender las situaciones presentadas dentro de su quehacer cotidiano. En suma se puede definir que la mayoría de los problemas que se suscitan en las aulas se pueden solucionar desde la práctica del docente. Una de las problemáticas con mayor presencia en las diferentes escuelas del país radica en cómo puede proceder el docente para atender lo establecidos en la curricular escolar sin dejar de lado las situaciones biológicas, psíquicas y sociales por ello que para dar soluciona las dificultades que se enfrentan dentro del aula se focalizó atenderlas a partir de la práctica docente desde un enfoque de investigación-acción. Sin embargo la falta de consolidación de los contenidos, aunado a la situación contextual de la realidad de los alumnos se decidió abordar la Pedagogía Crítica bajo la perspectiva de Paulo Freire .

Por lo anterior se sostiene el supuesto teórico: el docente que lleva una pedagogía crítica en el aula transforma su realidad y la de sus alumnos, favoreciendo el desarrollo del pensamiento crítico coadyuvando su autonomía. 
Como pauta a seguir, el documento se ha dividido en tres apartados, concusiones y referencias. El primer apartado ha sido titulado La investigación como principio de transformación, continuando con La pedagogía critica dentro del proceso de enseñanzaaprendizaje, El desarrollo del pensamiento crítico en el aula para la autonomía del alumno.

\section{La investigación como principio de transformación}

La epistemología es la encargada de entender y estudiar cómo se lleva a cabo el proceso de la adquisición del conocimiento, dicho en otras palabras es en la cual se sostiene el origen del conocimiento en el ser humano. La investigación por su parte, propicia las condicione para que el conocimiento que se construya o reconstruya sea significativo y sistematizado. Desde esta perspectiva, se ha comprendido y trabajado la investigación que se expone en el presente. La investigación, es un proceso sistemático que permite el desarrollo de conocimiento en los individuos, para Stenhouse (1985) la investigación es una indagación sistemática y autocrítica, indagación puesto que se fundamenta en una curiosidad y deseo de comprender, es estable, sistémica dado a que estudia las interrelaciones entre sí, pero ¿por qué es autocrítica?

Lawrence menciona que la investigación puede tomar otro camino cuando intervienen en ella intereses entendiendo estos como las influencias que puedan tenerse por patrocinadores, corrientes políticas, economía y toda aquella situación que provoque sentimientos y pensamiento subjetivo. Es autocrítica, porque pone en juego la capacidad de decidir nuestro actuar, el ser crítico al investigar, es controlar nuestras acciones para dar un seguimiento verídico a la investigación.

Llevar a cabo un proceso de investigación implica poder hacer frete a la suma de cuestionamientos que se hagan sobre ésta, es tener los fundamentos de cada proceso llevado. Dentro de las ciencias sociales, específicamente en la investigación-acción, aquella parte del proceso que transforma o contradice el supuesto teórico formulado, debe ser entendido como un área de oportunidad para abordar desde otra perspectiva, dado a que en una investigación de este tipo los tropiezos son únicamente una posibilidad más para la mejora.
Freire ( 2006) señala que lo primero que se debe hacer para la trasformación es reconocerse como hombre o mujer inacabados, pues a partir de la curiosidad por conocerse comienza la motivación para transformarse. Por ello, que se sostiene que la investigación es el inicio de la transformación, pues la indagación sistemática sobre la realidad de estudio, permite ampliar los panoramas de acción para la mejora.

La visión de investigación expuesta con anterioridad tiene como base el enfoque de la investigación- acción (action reserch) pues sugiere reflexionar sobre el actuar y modificar las acciones en pro de mejora, el carácter central de ésta (Carr \& Kemmis, 2005) es la autoreflexión formada por ciclos sucesivos de planificación, acción, observación y reflexión esto indica una práctica infinita, propia de individuos que buscan la transformación de su pensar y su actuar.

Para un docente, ser investigador requiere ponga en juego cada recurso biológico, psicológico, social y cognitivo que posea, esto provoca que analice minuciosamente su realidad, como ser humano, como profesor, como compañero así como su accionar en ella, de forma que se lleve un seguimiento de ésta. Chávez (2005) señala que la investigaciónacción es un proceso sistémico que induce a los participantes a teorizar sus prácticas al ejecutarse de esta manera la práctica va convirtiéndose en una práctica científica, de lo contrario carece de validez, sin permitir una reflexión continua y por ende queda susceptible a fallas o vicios que pasan desapercibidos por quien los realiza.

Desde la perspectiva de Kemmis ( 1985) action reserch se comprende como una forma de indagación autorreflexiva que realizan los participantes en situaciones sociales en orden a mejorar la racionalidad y la justicia de sus propias prácticas, su entendimiento de las mismas y las situaciones dentro de las cuales ellas tienen lugar

En suma, la investigación permite la construcción del conocimiento, a partir del reconocerse como ser humano (hombre o mujer) y la curiosidad que surge de ese proceso. 
Por el estudio de la realidad en las distintas interrelaciones que pueda establecer en ella misma; es objetiva pues se aleja de todo interés; requiere de todas las herramientas y recursos con que posea el investigador, y aquel docente que lleve a cabo este proceso sistémico de su quehacer en el aula genera práctica científicas pues a partir de la autoreflexión mejora sus propias prácticas, las comprende y es capaz de reformular aquello que le sea necesario para continuar, trasformando su realidad y convirtiendo su acción docente en una pedagogía crítica pues logra la autonomía en su actuar.

\section{La pedagogía crítica en el proceso de enseñanza- aprendizaje}

Para que se lleve a cabo la adquisición de conocimiento existe un proceso entre el que enseña y el que aprende conocido como proceso enseñanza-aprendizaje. Dentro de las áreas de psicología y pedagogía son vocablos comunes, sin embargo al profundizar en ellos se puede analizar que ambos van más allá de uso común dentro de los textos o expresiones diarias, pues enseñar es proveer al ser humano de experiencias que le permita adquirir un conocimiento nuevo, esa adquisición puede ser desde la construcción, reconstrucción o cualquier forma que provoque un cambio de pensamiento en el ser humano.

La enseñanza puede ser pues, el cúmulo de experiencias propiciadas ya sean formal o informal, formal cuando es llevada a cabo en la escuela, con una base sólida en planes y programas, mientras que la informal será la que provea el contexto, la sociedad fuera de la institución escolar.

Por otro lado, aprender es el punto donde el individuo moviliza sus saberes previos y los integra a dicha experiencia provocando con ello surja una idea distinta a la inicial a través de la reconstrucción y construcción de un conocimiento nuevo en el aprendiz, es decir, un proceso de construcción de nuevos conocimientos sobre la base de los conocimientos actuales (Baxter \& Glaser, 1998)
Las ideas anteriores permiten expresar que enseñanza-aprendizaje es un proceso en el que interviene el papel del docente quien propicia las situaciones de experiencia, y el alumno quien construye un conocimiento nuevo, sin embargo, no sólo el alumno construye sino también el maestro pues en cada experiencia que el profesor propicie pone en juego sus experiencias previas, ese trabajo en conjunto con los alumnos reestructura saberes, obteniendo un conocimiento nuevo, por ello se dice que es reciproco.

Si bien es cierto, la intención de todo educador debe girar a propiciar para los individuos los ambientes y experiencias adecuados para la adquisición y reconstrucción de conocimientos, así como también tiene la responsabilidad de hacer cuanto pueda para que el alumno encuentre significado a lo que hace, motivándolo a analizar cada una de sus acciones y las consecuencias que éstas tiene en su realidad, pues cuando el individuo desarrolla la capacidad de entender que esa realidad es parte de él y él de la realidad. podrá decirse que ha desarrollado un sentido crítico en su persona.

Si lo que se intenta es la transformación de la práctica, y el desarrollo del pensamiento crítico en los alumnos, se requiere de una práctica crítica, donde el profesor sea capaz de ejercer un juicio propio hacia una o varias situaciones, ponga en juego sus habilidades, sus realidades, más nunca sus intereses, pues la función de este pensamiento es ser objetivo.

Profundizando en la concepción de pedagogía crítica es necesario analizar que es una corriente que examina la escuela tanto en su medio histórico como también por ser parte del resultado social y político. La variante radical de esta corriente, supone su interés en la transformación social, favorece de manera directa a la sociedad sometida. Bajo esas premisas, la pedagogía crítica intenta proporcionar al maestro investigador medios para comprender el papel que juegan las escuelas dentro de una sociedad dividida en razas, clases, géneros, políticas y filosofías (Mclaren, 1997) 
Un profesor que busca transformar no solo su práctica sino su realidad y con ello la de sus alumnos, analiza las experiencias de los éstos, los materiales, las políticas educativas, institucionales y sociales, con el fin de favorecer el aprendizaje, así como también guía sus acciones de modo que éstas influyan en los alumnos y los haga críticos de su realidad, y con base en ello se comience la transformación social.

Así pues, para propiciar un pensamiento y una práctica crítica se requiere indudablemente de la dialéctica, ésta ha sido interpretada a lo largo de la historia de distinta manera, la primera y una de las interpretaciones más conocidas es la del arte de dialogar, sin embargo dentro del proceso de investigación que se llevó, la dialéctica es concebida como un proceso de análisis y de reflexión de contradicciones intenta desechar las historias y las relaciones de los significados y apariencias aceptados, logrando así trazar interacciones desde el contexto a la parte, del sistema interno al hecho (Mclaren, 1998)Con el uso de la dialéctica dentro de proceso de enseñanza-aprendizaje el docente comienza a trazar las conexiones con el contexto, la práctica que realiza, la que quiere realizar para atender las necesidades de conocimiento de la sociedad que atiende para que busque su mejora.

Ahora bien, la dialéctica representa gran parte de la totalidad del pensamiento crítico, pues las relaciones de análisis que se hacen entre lo que es y lo que no es provocan la creación o recreación de juicios personales. En la pedagogía crítica la implicación de la dialéctica va encaminada a que el maestro propicie las acciones necesarias donde el alumno vaya adquiriendo una conciencia crítica de su realidad, por ello, debe ser consciente que cada una de sus acciones repercuten de manera directa en la realidad de los estudiantes y en esa micro sociedad que es el aula.

Así mismo, dentro del proceso de enseñanza-aprendizaje surgen posturas particulares entre los alumnos y de alumnos con docente, por ello que el proceso dialéctico viene a favorecer el sentido crítico de ambas partes pues como componentes de una micro sociedad crítica, es lógico que surjan contradicciones, sin embargo entra en juego las capacidades que como comunidad crítica se requieren, respetando ideas y posturas contradictorias, pero que son válidas.

ISSN 2523-2479

ECORFAN $^{\circledR}$ Todos los derechos reservados
Así como también se buscan unificar criterios para cumplir objetivos en común sin violentar la diversidad de ideas. Dichas posturas de la pedagogía crítica van encaminadas a las diferencias entre escolarización y educación, Henry Giroux (Giroux \& McLaren, 1989) como radical crítico hace referencia a las distinciones de la escolarización y la educación, la primera de ella la concibe como una forma de control social, mientras que la segunda la refiere a la transformación de la sociedad tomando en cuenta al estudiante como un ser activo que se compromete con su desarrollo y el de la sociedad.

Bajo dicho referente, la pedagogía crítica supone pues que el maestro debe poseer un sentido humano, crítico de su actuar, de su hablar y de su pensar, debe ser activo, pero sobre todo, debe poseer la capacidad para transformar su práctica en función a las necesidades de sus alumnos y de su contexto, sin ataduras a ideas necias e inertes, propias de los vicios del quehacer docente.

La escuela se convierte entonces en el área de oportunidad para la transformación de patrones mentales tanto en docentes como en los alumnos, a manera de contribuir en los cambios sociales para mejorar la calidad de vida la comunidad educativa, de la práctica docente. A manera de cierre, puedo sostenerse que la pedagogía crítica es radical, pues pretende en todo momento que tanto maestros como alumnos observen el trasfondo de la realidad en la que viven y adopten las acciones necesarias para una transformación bien fundada.

\section{El desarrollo del pensamiento crítico en el aula para la autonomía del alumno}

Investigar en el aula rompe con la educación conductista en la que se ha estancado por muchos años la educación, sin embargo, las múltiples reformas que ejecutan los Estados toman como base el ciudadano que pretenden sea parte de la sociedad, establecen los programas, los planes de estudios, los periodos vacacionales y ofrecen a los docentes estrategias con el fin de cumplir con los objetivos planteados, pese a que las brechas de lo que se propone con la realidad sean extensas. 
El seguimiento realizado para efectos de esta investigación tiene lugar con un docente frente a grupo de educación primaria y de docentes en formación que comenzó con el análisis de su contexto, reflexionando que la verdadera problemática que enfrentaba radicaba en su práctica, pues debía atender las necesidades de alumnos, aula, escuela, contexto. Detectó que las distintas adversidades le impedían cumplir con el programa curricular podrían ser atendidas desde la investigaciónacción, desde la perspectiva de McLaren, Carr, utilizando los principios que Freire propone como parte de la pedagogía crítica.

Romper con los esquemas preestablecidos dentro de los diferentes nieles educativos, provoca situaciones que no siempre benefician a los docentes, pues se rompe con la comodidad y los vicios que los centros educativos y la comunidad escolar vienen arrastrando, o bien con políticas y lineamientos que ya no son vigentes a la necesidades actuales. Es a partir de este punto donde el docente investigador comienza a poner en práctica su sentido crítico.

El desarrollo de esta investigación demostró que la pedagogía crítica va más allá de una simple estrategia que promueva el compromiso de la comunidad educativa, pretendió la transformación de los patrones mentales de los elementos humanos que intervienen en el proceso de enseñanzaaprendizaje con el fin de transformar sus realidades para elevar su calidad de vida.

Es claro que lograr una transformación de mentalidades pasivas a mentalidades críticas requiere de un proceso sistemático y lago, donde el docente sea congruente con la pedagogía que aplica, su actuar y su pensar pues el tiempo que el docente trabaja con los alumnos sólo marca el inicio de un proceso largo y de por vida, como lo marca el supuesto teórico que trajo la investigación al presente, donde se sostiene que el docente investigador que trabaja bajo una pedagogía crítica favorece el desarrollo del pensamiento crítico, pensamiento que transforma al individuo y éste a sus distintas realidades, coadyuvando a la formación de convicciones.
El seguimiento realizado a esta investigación ha sido de ocho años, ocho en el nivel de educación primaria y seis en la formación de docentes, los hallazgos han sido tan variados como el número de grupos que se han atendido, sin embargo, el punto en común en ellos radican en lo siguiente. Con lo que refiere a la práctica realizada por el docente, este requiere de un plan de acción que satisfaga las necesidades de los colectivos, tomando en cuenta que él es parte del colectivo.

El inicio de cambio de las realidades mediante la investigación con los alumnos, se dio mediante el análisis constante de las acciones ejecutadas, con base en ello, se reformulaban de modo que atendieran las necesidades que se presentaban., esto permitió valorar la práctica docente, enseñar a pensar y aprender a pensar no ha sido un proceso fácil, pues el docente debe ser consciente que el proceso de construcción de conocimiento tiene varias implicaciones, como: disposición y voluntad de aprender, llevar un seguimiento verídico, alejarse de autocompasión o justificación ante situaciones desfavorables, observar y analizar la rapidez con la que puede modificarse una realidad y percatarse de los vicios que en ella surjan para atenderlos, entender que el proceso de construcción del conocimiento varía según las características del ser humano y de éste con sus contexto y por último que los individuos que comienzan a pensar críticamente pensarán de manera distinta al resto de la sociedad y deberán estar preparados para lo que puedan enfrentar.

Cuando el docente pone en práctica lo anterior, comprende que la pedagogía crítica es también humanista, y requiere poner en juego la capacidad para evaluar los procesos de los alumnos, la empatía con éstos y con quienes integran las diversas realidades, la capacidad de diálogo para la solución de conflictos, la práctica de una dialéctica constante, todo ello va provocando que el maestro cambie sus estructuras mentales y adopte conductas congruentes no solo en su quehacer docente, sino en su vida, es decir, logra ser un individuo autónomo con convicciones que transforma sus realidades y por ende la de los alumnos que atiende. El alumno que desarrolla un pensamiento crítico logra mejorar su aprovechamiento académico, mejora sus relaciones personales, familiares y sociales. 
Aprende por convicción e indaga aquello que desea, decide bajo sus términos siempre teniendo en cuenta que es responsable de cada decisión y acción realizada, enfrentado las consecuencias que estas traigan con seguridad y fundamentación. Se vuelve un ser emocional y académicamente estable.

Durante el proceso de ejecución del plan de acción el docente en cuestión, ha enfrentado una serie de dificultades relacionados con las ideas distintas de los centros escolares, las prácticas viciosas en ellos, la poca capacidad de las instituciones para atender a alumnos pensantes, la restricción a la aplicación de actividades.

Ahora bien, la evaluación bajo una pedagogía crítica, implica poner en práctica el juicio de los alumnos, de ahí que se han utilizado distintas modalidades de evaluación como la coevaluación, la autoevaluación y la heteroevaluación en ambos niveles de educación. Cada una de ellas, han permitido poder apreciar las perspectivas de los alumnos, con base a dichas evaluaciones se realizan modificaciones en el plan de acción y permiten un seguimiento genuino del presente.

A manera de conclusión, dentro de los beneficios que trae el trabajar con una teoría crítica para favorecer la autonomía está que propicia la formación y modificación de hábitos, pues en ese proceso de transformación de patrón mental, los alumnos mejoran su calidad de vida, sus relaciones personales, su rendimiento académico, su relaciones familiares, factores determinantes que guiaron su acciones, pues comprenden que cualquier acción que realicen en cada una de sus esferas (familia, amigos, escuela,) de manera positiva o negativa tienen sus repercusiones, cuando logra lo anterior se sostiene que el alumno es autónomo.

\section{Conclusiones}

En suma, la pedagogía crítica busca la trasformación de la realidad a partir de juicios claros y objetivos, que desarrollen en el alumno cambios de hábitos en todos los que integran la realidad, fomenta las relaciones personales sanas, favorece el desarrollo de las habilidades lingüísticas, permite que los contenidos en clase sean significativos para los alumnos e incrementa el rendimiento escolar.
Determina que el alumno genere ideas nuevas, las proponga, ejerza una crítica, misma que le permite ampliar sus horizontes y los de sus compañeros, logrando con ello una autonomía, pues sus decisiones se basan en convicciones.

Así mismo, trabajado con investigaciónacción, permite observar las debilidades y fortalezas de la práctica, así como también fomentar un ambiente armónico y crítico con los alumnos, los padres de familia, la investigación acción pues, no solo transforma la práctica educativa sino la perspectiva de la educación, ya que orienta que el docente profundice en su sentido humano, rescate y ponga en prácticas valores, y sea capaz de proponer las modificaciones desde las aulas hacia afuera, pues como lo sostiene Jimeno Sacristán, en el maestro quien realimente conoce las necesidades curriculares pues es quien la ejecuta.

El alumno logra ser autónomo cuando posee un pensamiento crítico y es capaz de orientar su actuar en pro de una mejor calidad de vida, discrimina información, indaga en lo que necesita, formula sus propios procedimientos a través de convicciones, analiza desde distintos enfoques su realidad, es capaz de modificar su conducta, de autorregularse, argumenta sus posturas, respeta las ideas de otros, practica valores y se rige dentro de la ética, es empático, busca el bien común y cuestiona para aprender.

Como continuación de ésta investigación, se pretende dar seguimiento a los docentes en formación con los que se ha trabajado esta propuesta, con la finalidad de sistematiza cómo es su actuar en las aulas de educación básica, y observar hasta qué punto la propuesta les proporciona las herramientas para cumplir con la función liberadora de la educación.

Por último y bajo las concepciones de la investigación acción, continuará el seguimiento cíclico que permita un avance mayor en las prácticas de los docentes interesados en trasformar sus prácticas para beneficiar a la sociedad, desde lo que sí está en sus manos y de ellos hacia el contexto. 


\section{Referencias}

Chavez I.,(2005). Liderazgo +participación $=$ autonomía escolar. Una experiencia de investigación acción en las escuelas de Colima, $1^{\text {a }}$ edición, editorial colección editorial voluntades, Morelia, México

Baxter, G. P., \& Glaser, R. (1998). Investigating the cognitive complerrity of science assessments. Educational Measurement: Issues and Practice. https://doi.org/10.1111/j.17453992.1998.tb00627.x

Carr, W., \& Kemmis, S. (2005). Staying critical. Educational Action Research. https://doi.org/10.1080/09650790500200296

Freire, P. (2006). Pedagogía de la autonomía. Saberes necesarios para la práctica educativa. In Educación y Territorio.

Giroux, H. A., \& McLaren, P. (1989). Critical pedagogy, the state, and cultural struggle. In Teacher empowerment and school reform.

Kemmis, S. (1985). Action research and the politics of reflection. Reflection: Turning Experience into Learning.

Mclaren, P. (1997). Critical pedagogy. International Journal of Phytoremediation. https://doi.org/10.1080/1047621970090101

Mclaren, P. (1998). Revolutionary Pedagogy In Post-Revolutionary Times: Rethinking The Political Economy Of Critical Education. Educational Theory. https://doi.org/10.1111/j.1741-

5446.1998.00431.x

Stenhouse, L. (1985). El Profesor Como Investigador. In Investigación y desarrollo del curriculum. 